\title{
Enabling ubiquitous sensor-assisted applications on the internet-of-things
}

\author{
Stefan Forsström • Theo Kanter
}

Received: 30 November 2012/ Accepted: 8 April 2013/Published online: 24 August 2013

(C) Springer-Verlag London 2013

\begin{abstract}
The increase in sensor-assisted applications such as sensing campaigns and interactions in large-scale populations put new demands on seamless sharing of sensor information on a global scale. Existing support is limited due to simplified information models reflecting narrow application scenarios and poorly scaling architectures. In response to this, we present an application architecture for pervasive internet-of-things applications which circumvents these limitations. Our architecture enables applications to utilize information from sensors and wireless sensor networks via a peer-to-peer overlay. The overlay shares sensor information in an generic information model, which is extensible and thus enables intelligent application behavior. Our evaluation of the architecture indicates that it supports the sharing of sensor information on a global scale in sensor-assisted applications, with low response times. Finally, we evaluate the feasibility of running such applications in end devices with limited resources in a sensor-assisted application prototype.
\end{abstract}

Keywords Internet-of-things - Context awareness · Ubiquitous

S. Forsström ( $\square)$

Department of Information Technology and Media,

Mid Sweden University, 85170 Sundsvall, Sweden

e-mail: stefan.forsstrom@miun.se

T. Kanter

Department of Computer and System Sciences,

Stockholm University, 16440 Kista, Sweden

\section{Introduction}

With the current escalation of smart mobile devices, people demand applications to behave more intelligently. These applications are integrated into people's everyday lives and are made aware of their user's situation and context, in order to change their own application behavior. This opens up a new field of user friendly services, which can pervasively be per person adapted to provide the best possible service for that particular user. In detail, these contextaware applications have enable us to use the features of mobile computing and intelligent reasoning in new forms of applications [1], such as sensing campaigns and interactions in large-scale populations. These types of features are also included in the vision of an Internet-of-things [2], where the expectations are in the order of 50 billion connected devices by 2020 [3]. For example, using smartphones to gather sensor information and create contextaware mobile applications, which also can utilize sensor information from other connected devices. In this paper, we present a new approach to context-aware applications in ambient environments and for the Internet-of-things, such as applications utilizing real-time sensor sharing between different devices, large-scale crowd sourcing of sensor information, and per person adapted services.

Current context-aware applications focus on a quite narrow scenario, where they can manage the communication without scalability issues. This has led to a large proliferation in the area that has obscured the real problems faced with large-scale deployment. These early successes include for example health care solutions [4], which can share simple data such as heart rate and blood pressure a few times per second, and location-based services [5], which often claim to be operating in real time even when they only update the location every few seconds. Smart home 
solutions such as [6] focus on local exchange and reasoning of contextual information, but hiding their limited potential when sharing with all connected entities on a global Internet-of-things. We foresee that the future will demand better services in the form of faster update frequencies, awareness, and reasoning. Thus, applications will require a continuous stream of sensor and actuator updates for their regular operation. Furthermore, as our surroundings become more aware through an increasing amount of sensors, the amount of contextual information on the Internet-of-things will also increase. In response earlier shortcomings in handling this vast amount of information, we are proposing a solution which is based on a fully distributed system and provide real-time sensor information delivery. Our solution is an application level platform that provides peer-to-peer distribution of sensor information in a reliable way among connected entities, which also has an extensible information model that enables intelligent application behavior. The platform can thus be used to create an Internet-of-things, where any device that is running the platform may connect with other peers and share information in an efficient way. This also includes mobile clients running on devices with limited resources, such as smartphones.

\subsection{Scenario}

The scenario and problem that is studied in this article is explained by following Sarah around in her daily life: Sarah lives in an urban environment and has connected her sensors with her health center. When she wakes up in the morning, her smartphone suggests a menu for breakfast, based on her current medically suggested diet. After breakfast, she receives information concerning meetings at the office, and the current traffic information indicates that it is necessary to leave a little earlier than usual. She is also notified and suggested to take a warm jacket, because it is colder than usual outside. While driving, she receives realtime notifications on the shortest possible route to reach her office. Furthermore, when arriving at the office, she is alerted on the pending work and agenda. During the work day, wearable health sensors indicate that she needs to update her health profile at the health center and see a doctor, and she thus retrieves a suggested appointment depending on her work schedule and availability. After visiting the doctor, her smartphone retrieves the updated information about the new medications and diet plans. When traveling back home, she receives information regarding a pharmacy on the way with her new medications available. After leaving the pharmacy, she is also informed about offers regarding groceries from the supermarket next to the pharmacy, which matches her new diet plan. Upon arrival at home, the events for the following day are available and her enriched connected life continues.

\subsection{Concrete goals}

To address the problems Sarah faced in the stated scenario, we have identified six concrete goals which must be met by a proposed solution. Thus, to successfully enable ubiquitous sensor-assisted application on the Internet-of-things, the following goals must be met:

1. Sensor-assisted applications must have seamless access to global sensor information in a ubiquitous manner.

2. The sharing of global sensor information between end devices must occur in real time and without any unnecessary delays.

3. The sensor information sharing must scale well with a vast number of connected entities, thus also avoiding any central points of failure.

4. A generic and extensible information model is needed in order to enable intelligent application behavior.

5. The information sharing must also support discovery, querying, and searching for new sources of information.

6. End devices must be able to balance their resource consumptions against quality-of-experience requirements on a per application basis.

The remainder of this paper is focused on answering these goals and is organized in the following way: Sect. 2 presents related work in the area. Section 3 presents our proposed architecture and approach. Section 4 presents how we extend the existing MediaSense platform to realize the proposed approach. Section 5 presents our proof-ofconcept prototype and an evaluation of it. Section 6 presents an evaluation on the feasibility of different resource limitations for the quality of experience in applications on the Internet-of-things. Finally, Sect. 7 presents our conclusions and future work.

\section{Related work}

Context-aware applications have been studied for some time, but with the recent escalation in the mobile market, they have proliferated and increased in multitude. The constant change of context information dictates that it must be gathered from many different sources concurrently at the same time. This is often achieved by using automated sensors, predefined personal profiles, intelligent scheduling, statistics gathering, etc. Furthermore, the recent advances in this area have so far produced a large number of different platforms for creating context-aware applications. These related architectures can be organized into three distinct categories, depending on where they store the actual context information. In detail, these three categories 


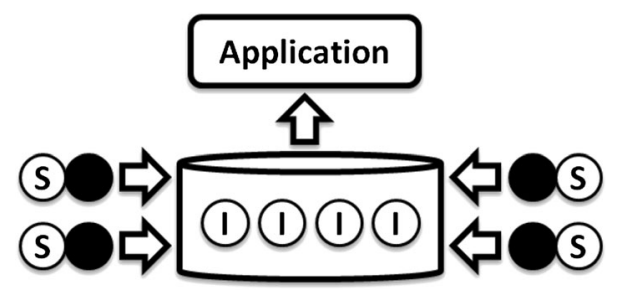

Fig. 1 Overview of typical centralized architectures

are as follows: centralized, semi-distributed, and fully distributed architectures.

\subsection{Centralized architectures}

Centralized architectures store the context information under a single administrative authority, either in a single large database or replicated in a cloud-based manner. An example on how this can look like can be seen in Fig. 1. In this figure, we have the end devices denoted as black dots, sensors denoted as $S$, and the actual usable information denoted as $I$. In detail, this figure shows how the sensors are connected to the devices which simply send all the information to a centralized point, which applications in turn communicate with. Related work examples of this type of centralized storage include SenseWeb [7], SENSEI [8], and SicsthSense [9]. Where for SenseWeb, for example it uses a hierarchical triangular mesh indexing scheme implemented as tables a SQL backend server, SENSEI stores their information in a so-called resource directory that they have implemented using Java and a MySQL database, and SicsthSense stores the information in a cloud-based manner accessed using REST calls. However, all of these centralized architectures have scalability issues when the number of updates and queries increases in magnitude. Therefore, they will have problems with support for continuously updating data with low latencies. These architectures are also prone to failure, because they expose single points of failure.

\subsection{Semi-distributed architectures}

Semi-distributed architectures store the context information locally on each entity in a peer-to-peer manner, but still maintain a centralized authority for the exchange of context between peers. An overview figure of this can be seen in Fig. 2, which shows how the information $I$ from the sensors $S$, now is stored on the end devices (the black dots) but still brokered by one or many centralized components. Typical for these architectures is that they use sessionestablishment protocols to exchange the context, but under supervision by a centralized authority. Mobilife [10], CONTEXT [11], and ADAMANTIUM [12] are examples

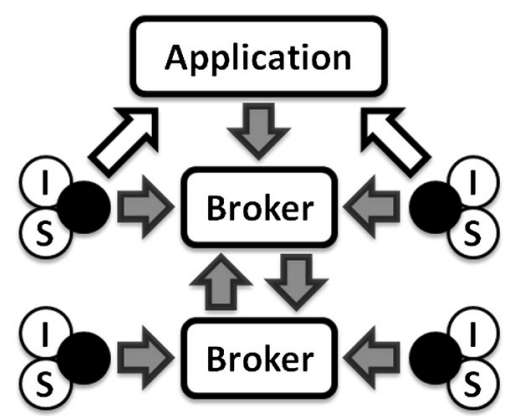

Fig. 2 Overview of typical semi-distributed architectures

of such systems. Where ADAMANTIUM follows this typical example and uses the SIP protocol [13] to establish sessions. These architectures scale better in comparison with centralized architectures, but they still maintain a centralized component. This will become a bottleneck for the context exchange when the entities perform many queries on a large and continually changing dataset, since the centralized component has to administrate all the sessions, even if the actual exchange is performed outside of the centralized component.

\subsection{Fully distributed architectures}

Fully distributed architectures both store and administrate the context locally on each entity in a peer-to-peer manner. An overview of this can be seen in Fig. 3, which shows how all the useful information $I$ from the sensors $S$, is stored on the end devices (the black dots), as well as the being exchanged in a pure peer-to-peer manner. Typical for these architectures is that they often utilize Distributed Hash Tables (DHT) to enable logarithmic scaling when the number of entities increases in magnitude. Examples of such architectures are SOFIA [14] and COSMOS [15]. Naturally, these systems do not contain any single point of failure and are thus more resilient, even if the distribution itself often require additional overhead for maintaining an overlay. The main problem of fully distributed architectures is that it puts a larger responsibility on the end devices. These end devices can however be limited in capacity, e.g., bandwidth and processing power, which will induce issues when sharing context information on a large scale.

\section{Our proposed architecture}

Different locations, people, and things have been taken into consideration in the scenario, for example Sarah, her home, the office, and a hospital. In our proposed architecture, we see all of these as more than just different end devices with 


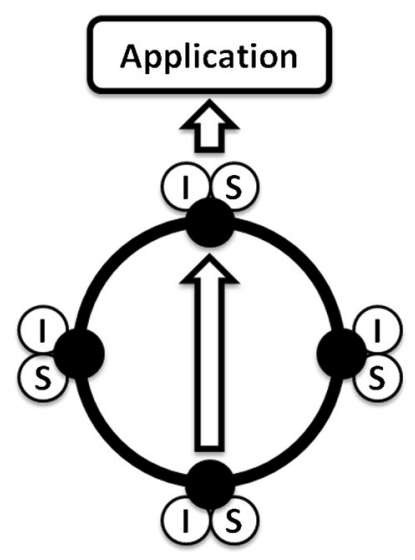

Fig. 3 Overview of typical fully distributed architectures

attached sensors, and we see them as different individual entities. An overview of our proposed architecture with these connected entities can be seen in Fig. 4. In detail, the figure shows multiple entities (with attached sensors denoted as $S$ and actuators denoted as $A$ ) connected through a common communication architecture with a supporting back-end system. The idea is that all connected entities shall be able to communicate with all other entities in a fully distributed manner, but have access to different supporting systems for resource heavy tasks such as persistence and time-consuming computations. In comparison with related work, our architecture should be more reliable and well scaling than centralized and semi-distributed architectures, while still being very lightweight when compared to other fully distributed architectures. This is because we will combine the benefits of scalability and speed from fully distributed architectures, as well as providing support for services that require features which fully distributed architectures traditionally have had problems to achieve, such as persistent storage.

\subsection{Context entities}

As previously stated, we see the persons, things, locations, etc. from the scenarios as different context-aware entities. In detail, we consider any entity that have sensors or actuators attached to itself and can communicate this information forward, as an entity. All these entities will have a digital representation of its current situation, a form of self-perspective, which we call a context schema. This context schema contains all relevant knowledge for a particular entity's current situation and context. In detail, the schema is the composite set of all local contextual information and all relevant contextual information from other entities. This can be seen in Eq. 1, where $C_{n}$ is the resulting context schema for an entity $n$ containing a set of information $I$. Where $I_{n}^{\text {local }}$ is the set of local information

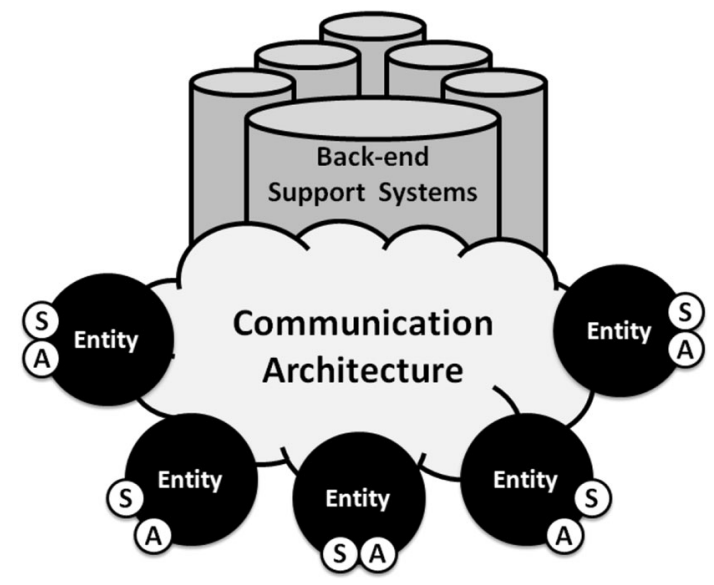

Fig. 4 General overview of the proposed architecture

available at entity $n$, and $I_{n}^{\text {relevant }}$ is the set of all relevant information for entity $n$ from other entities. In detail, Eq. 2 defines $I_{n}^{\text {local }}$ as the set of all information on the local entity $n$ for all different information types $k$. Equation 3 shows how we calculate the set of relevant context information from other entities. For all other entities $m$ and for all their $k$ different information types, $i_{m, k}$ is the information being evaluated and $f_{k}^{\text {relevant }}\left(i_{m, k}\right)$ is the relevance function for determining whether the information of type $k$ from node $m$ is relevant or not. In which, $x_{n, k}$ is the threshold value for the relevance evaluation, given by the evaluating entity $n$ for the particular information type $k$. The idea is thus to modify $x_{n, k}$ to fit the desired quality, precision, complexity, etc. for a particular entity's application scenario.

$$
\begin{aligned}
& C_{n}=\left\{I_{n}^{\text {local }} \cup I_{n}^{\text {relevant }}\right\} \\
& I_{n}^{\text {local }}=\forall_{k}\left\{i_{n, k}\right\} \\
& I_{n}^{\text {relevant }}=\forall_{m \neq n} \forall_{k}\left\{i_{m, k} \mid f_{k}^{\text {relevant }}\left(i_{m, k}\right) \leq x_{n, k}\right\}
\end{aligned}
$$

In our proposed architecture, these context schemes for the context entities are filled with information from a large amount of entities with very rapidly changing sensor information. Where we expect to produce more information than any end application is capable of managing. But the sought after quality of experience and available resources of the end applications will be a factor for the contextual relevance evaluation, and thus, the information overload should be hidden for the end user when interacting with the application. It is however very important that the user still feels satisfied with the quality of the presented information in the application. This approach does however rely on the existence of a method for evaluating context relevance. Such functions have been studied in many forms [16, 17], for example by using finite state machines, Markov models, etc. But performing these calculations will be very time-consuming or sometimes 
even impossible to calculate, such in the cases of nonlinear or multidimensional values. This is why the problem is often simplified in many related implementations to only manage simple context values, such as latitude and longitude.

\subsection{Communication architecture}

Sarah interacts with quite many entities in the stated scenario. Thus, the communication architecture should provide the means for the real-time information exchange and support for a wide range of applications, while avoiding any central points of failure. Where we believe that the only true way of enabling real-time capabilities is to directly exchange data between entities, with no proxying or middle management. Hence, in order to address this, some form of fully distributed architecture will be needed. Furthermore, it should be capable of peer-to-peer exchange to minimize the delay and be lightweight in order to run on limited devices. The supporting architecture should also be transparent and pervasive to the user, so that the user can focus on what is important, i.e., their own life and the benefits from the services that they are using.

\subsection{Back-end support systems}

The complexity of the services which Sarah is interacting with requires more functionality than any personal device can possess, for example reliable persistence of her information, historical sensor values, computational heavy reasoning, billing, and privacy protected information. These types of services are very difficult to handle in a fully distributed manner with resource-limited devices. Thus, we envision that there are nodes with higher responsibilities and better resources connected to the communication architecture as well. We call these support systems, and they act as a back end to different applications, which might require additional resources for various reasons. For example, Sarah's hospital will probably use a back-end support system for the health records, which must be stored persistently and securely. In similarity, her office might also use a back-end system for the company's internal information and protected resources.

\section{Realizing the architecture}

In this paper, we realized the general architecture using the MediaSense platform, which gave us a solid framework for performing evaluations, testing, and field trials. In detail, the MediaSense platform is an open source peer-to-peer Internet-of-things platform for distributed sensor and actuating, in a scalable manner without any central point of failure. It is however important to note that our proposed architecture only utilize the basic functions of MediaSense to communicate and connect entities, and all new features proposed in this article were realized on top of the existing platform. The platform itself has been updated in several stages $[18,19]$, but it is now released to the public as an open source project and can be used in a wide range of applications. Our realization can be seen in Fig. 5, and it shows the peer-to-peer platform connecting entities, which are denoted as black circles in the figure. Furthermore, the MediaSense platform supports both sensors and actuators, which are denoted as $S$ and $A$ in the figure. The idea is then as such that MediaSense realizes the communication architecture component from Fig. 4.

\subsection{Architecture layers}

The MediaSense platform uses a five layer architecture, i.e., interface layer, sensor/actuator layer, add-in layer, dissemination layer, and networking layer. A figure of the layered MediaSense architecture can be seen in Fig. 6.

The interface layer is the public interface through which applications interact with the MediaSense platform. Thus, all applications are connected to this layer and are using the publicly exposed API. In detail, the interface layer contains the MediaSense application interface, which is a generic and standardized application programming interface (API) for developers to build their own applications upon.

The sensor/actuator layer is the layer containing the physical sensors and actuators. Where they connect into the MediaSense platform via the interface layer. The purpose of the sensor/actuator layer is to enable a generalized

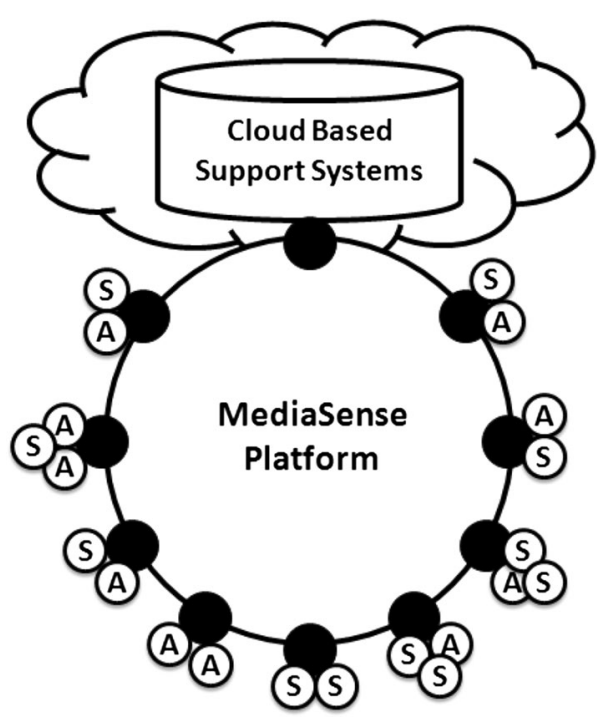

Fig. 5 Overview on the realized architecture using the MediaSense platform 


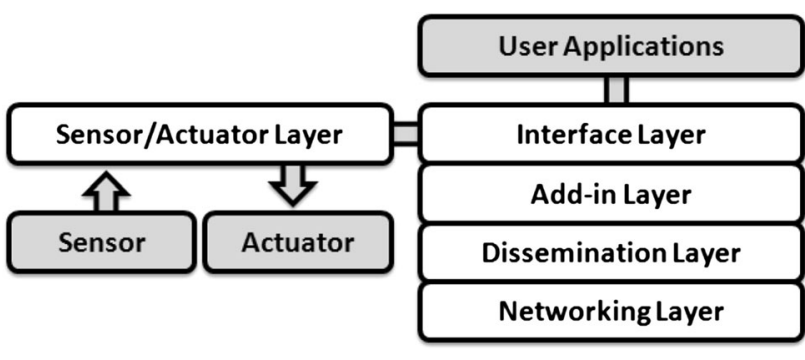

Fig. 6 The MediaSense platform's layered architecture

method to produce and consume information in the MediaSense platform. The problem is that there exist a large number of different sensors and actuators, which use many different hardware technologies. Thus, this layer provides a generic mean of connecting any type of sensor and actuator into the platform in a seamless manner.

The add-in layer is for adding extra functionality to the MediaSense platform. This is done by creating and activating an extension component, such as publish/subscribe functions, caching, optimizations, security, authentication, reasoning. The purpose of the add-in layer is to enable developers to add optional functionality optimization algorithms to the MediaSense platform. Thus, the add-in layer makes it possible for the MediaSense platform to use tailored extensions to meet specific application requirements, which can be loaded and unloaded in runtime when needed.

The dissemination layer is responsible for disseminating information to different peers using the Distributed Context eXchange Protocol (DCXP) [20], thus providing the primitive functionality of the system. The dissemination layer is also in charge of managing the connections to all other entities in the fully distributed system. The dissemination layer contains a lookup service which is used to find and resolve locations of information within the system, utilizing a Distributed Hash Table (DHT). In detail, DCXP resolves a so-called Universal Context Identifier (UCI) in the DHT and can then subsequently transferring information directly to and from the resolved entity in a peer-topeer manner. In detail, the current implementation uses a DHT where all entities are connected in a circular manner, similar to the Chord DHT [21]. The peer-to-peer traffic uses a reliable UDP protocol which does not have any initial handshaking and thus can deliver the actual useful information in the first packet, as well as resend any lost packets. Thus, the dissemination layer enables the real-time dissemination of information between all entities that participate in the system, because of the peer-to-peer nature of the communication.

The networking layer is the lowest layer and signifies the underlying IP network infrastructure, which the MediaSense platform is operating on. The platform is designed

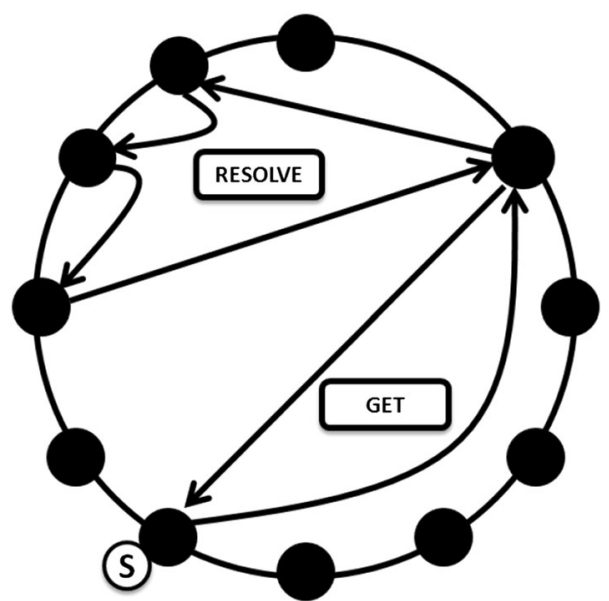

Fig. 7 The MediaSense platform's signaling for resolving and retrieving sensor values

to operate over heterogeneous infrastructure, including wireless and mobile. Thus, the purpose of the networking layer is to solve any problems of connecting and communicating over the current underlying network infrastructure.

\subsection{Communication and signaling}

The communication and signaling algorithm in the MediaSense platform is quite simple. The underlying DHT manages and takes care of the relations to other connected entities. Any user can call the interface layer to register a sensor name within the system, and then, any other user can resolve this sensor name in order to find the end point responsible for that sensor. The user can then subsequently retrieve the latest sensor value from the source. In detail, the user performs the register UCI function inside the lookup service, which stores the location of the registering entity inside the DHT. Thus, from this moment onwards, any other user can resolve this UCI with the resolve function to find the originating entity. Following this, the user can create a peer-to-peer session with the originating user and get the sensor information directly from the source. A figure over this resolving and retrieving can be seen in Fig. 7, which shows the initial resolve and the following peer-to-peer session. In detail, the figure shows how a node can resolve a UCI within the MediaSense system with logarithmic lookup time and subsequently retrieve the actual sensor value directly from the other entity which has the sensor attached.

From this, we have also analytically analyzed the estimated response times of our system. Where $n$ is the total number of entities in the system, $t_{\text {transmission }}$ is the time for the communicating between two entities, and $t_{\text {lookup }}$ is the time for a single lookup in the local partition of the hash table on a node. The estimated maximum latency to 


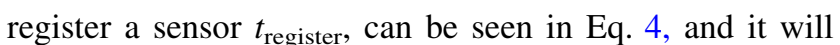
logarithmically scale with the size of the network. The estimated maximum latency for a resolve $t_{\text {resolve, can be }}$ seen in Eq. 5, and it follows the same scale as the register, but has an additional delay for the response to return back. The estimated maximum latency for retrieving a sensor value $t_{\text {retrieve }}$ can be seen in Eq. 6, given that we already have performed the resolve beforehand, which simply is the communication delay to and from the two entities. It is however important to note that the value of $t_{\text {transmission }}$ highly depends on the links between which the entities that are communicating.

$$
\begin{aligned}
& t_{\text {register }}=\sum_{i=1}^{\log (\mathrm{n})}\left(t_{\text {transmission }}+t_{\text {lookup }}\right) \\
& t_{\text {resolve }}=\sum_{i=1}^{\log (\mathrm{n})}\left(t_{\text {transmission }}+t_{\text {lookup }}\right)+t_{\text {transmission }} \\
& t_{\text {retrieve }}=t_{\text {transmission }}+t_{\text {transmission }}=2 \times t_{\text {transmission }}
\end{aligned}
$$

\subsection{Cloud-based back-end support systems}

Because of the limited resources on the devices of the entities, we have created additional extensions to the $\mathrm{Me}$ diaSense platform to support cloud-based back-end support systems. Hence, this have been created as a new feature for MediaSense and is thus a new contribution to the platform. We did this in order to correctly handle sensitive information, redundancy, reasoning, billing, security, etc. We needed these features in order to fulfill the high demands on ensuring that no vital information is lost and that the correct decisions are made. In detail, our extension uses a cloud server architecture, for example gather the values from the distributed entities in the system. In detail, this provides additional storage of historical information in case of failure and the ability to provide computational heavy reasoning based on this gathered data. This cloud server also has an additional security component, which only allows users with the correct privileges to access certain private information in order to protect privacy. This extension to the MediaSense platform also provides means of running traditional Web service applications, which can utilize the distributed sensor sources. However, this cloudbased back end endangers the real-time aspects and should not be used in scenarios, which has high demands on realtime delivery, but it should be used when intelligent and computational heavy decision making is required. Thus, the cloud-based back end should only be seen as a complement to the normal peer-to-peer exchange of the MediaSense platform.

\subsection{Discovery of relevant information}

In the stated scenario, there is a fair amount of unknown information which must be discovered, ranked, and determined what is important to the current application. For example, as Sarah travels, she might find new sensors from users, which she had no prior knowledge about, and these new sensors must be evaluated based on importance and how relevant they are to her current situation. This also includes evaluations of reliability, for example a temperature sensor close to a hot radiator is probably not the best sensor available, because it will report a temperature several degrees higher. In response to this, we were required to extend the basic MediaSense platform even further, in order to address these problems. We have solved this by both allowing the end devices themselves to either perform computational heavy reasoning or to offload the responsibility to a back-end support system, which has more powerful hardware for complex computations.

Multiple statistical methods exist to determine the most relevant information and to discover new interesting entities. One possible approach was explained in Eqs. 1, 2, and 3 , which uses a predefined relevance threshold based on the application scenario and type of information being evaluated. Another possible approach we are exploring is to filter the information based on the current situation. As each entity has a context schema of its own self-representation, the schema can also be used to find the best possible fit into a set of predefined situations or states. We can see this as a Markov model, to create probabilities of belonging to a certain state and the transition between them. From the scenario, we can for example build up a Markov model like in Fig. 8, where we can see how Sarah can transit between the predefined situations with a certain probability. But in a larger and more general case, the problems are apparent. This is because the amount of possible states increases in magnitude for the general case, perhaps even to an infinite degree. Thus, we have no way of classifying all the possible states, and the calculations will be very resource consuming. However, there are approaches which apply hidden Markov models to solve the problem in the general case, given that we have a large enough dataset of what is considered being relevant contextual information. We can also aid in the discovery of relevant information by ranking information based on the importance and reliability of their sensor source. Given the fact that many entities trust and use the same sensor source, should indicate that it is a good source of relevant information, especially in combination with the current situation from the Markov model and the context schema information relevance evaluation. 
Fig. 8 An example Markov model based on the scenario

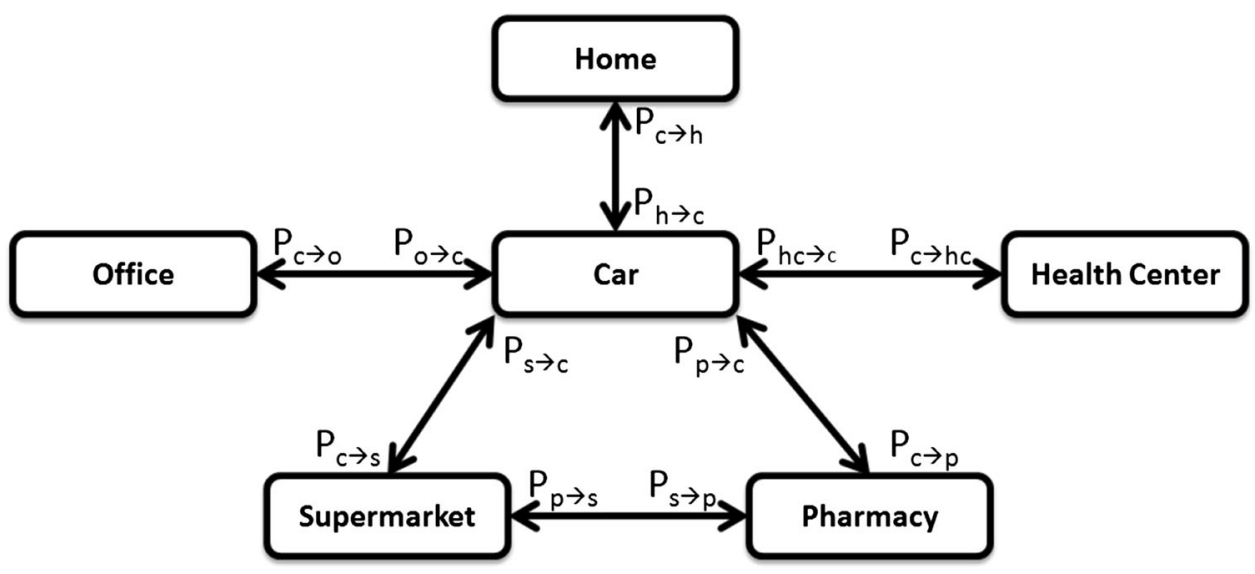

\section{Proof-of-concept application}

Many proof-of-concept applications have been created in order to verify the realized architecture. But in this paper, we focus on a particular proof-of-concept application, which was based on health care services, similar to the services that Sarah used in the scenario. In detail, our proof of concept has features such as health monitoring, appointment reservations, historical graphs, suggested diet plans, id tag scanning, location tracking, and alarming an emergency. However, the intelligence logic behind these features was quite simple. The application was demonstrated on three different devices in order to show the versatility of the platform and the application areas. They are in detail: an Android smartphone, a TV system, and a normal Web interface. Figure 9 shows sample views of these three interfaces.

Preliminary measurements of latencies and response times have also been done on the proof-of-concept applications. We measured two parameters: resolving a sensor name in the system and retrieving a value from a remote user. In detail, the resolve was measured from the resolve call on the interface layer, to when the resolve is returning the answer with the location of the sensor. Following the same setup, the retrieve was measured from the get call on the interface layer, to when the newest value is returned back to the application. We performed the evaluation between a smartphone and a computer connected to the same WiFi, with 10 more nodes connected to the DHT at the same time. The actual measurements were performed on the smartphone side, and it was tested on a Samsung Galaxy Nexus smartphone. In detail, the results we got were that the resolve operation took on average $70 \mathrm{~ms}$ with a standard deviation of $77 \mathrm{~ms}$. While the average for the retrieve operation was $53 \mathrm{~ms}$ with a standard deviation of $61 \mathrm{~ms}$.

We also did a preliminary evaluation with more entities in the system, this in order to get an indication on scalability. But because of the replications in the DHT to achieve the logarithmic lookup, no major difference could be observed. In detail, we performed the same test with 500 nodes in the system, and we got a response time for resolving on average $69 \mathrm{~ms}$ with a standard deviation of $66 \mathrm{~ms}$. We also got a similar response time for the retrieve operation, namely $51 \mathrm{~ms}$ with a standard deviation of $70 \mathrm{~ms}$. These measurements can however only be used as a preliminary indicator for large-scale deployment because of the limited number of available physically connected sensors and devices, and the fact that this was done over a local WiFi. But our initial testing indicates that the resolving (and thus also the registering) should increase logarithmically in delay with the amount of connected entities. The retrieval response time should however stay the same, because it uses peer-to-peer traffic and is thus agnostic of the size of the network. Our initial measurements also seem to correspond quite well to formulas 4,5 , and 6 from Sect. 4.2.

\section{The feasibility of ubiquitous sensor-assisted applications}

In this section, we will summarize our findings into the potential feasibility of sensor-assisted applications with a high quality-of-experience demand, when running on resource constrained devices. These resources can for example be battery, data link speed, computational power, etc. The early successes of application based on Internetof-things ideas have hidden many of the future issues related to these limited resources, as they have focused on a limited scenario and small-scale deployment. But we believe that the resource demands of future applications will increase in magnitude as they seek global proliferation and large-scale deployment, especially as the demands for more intelligent applications will increase. However, we know that the available resources on the devices will also 

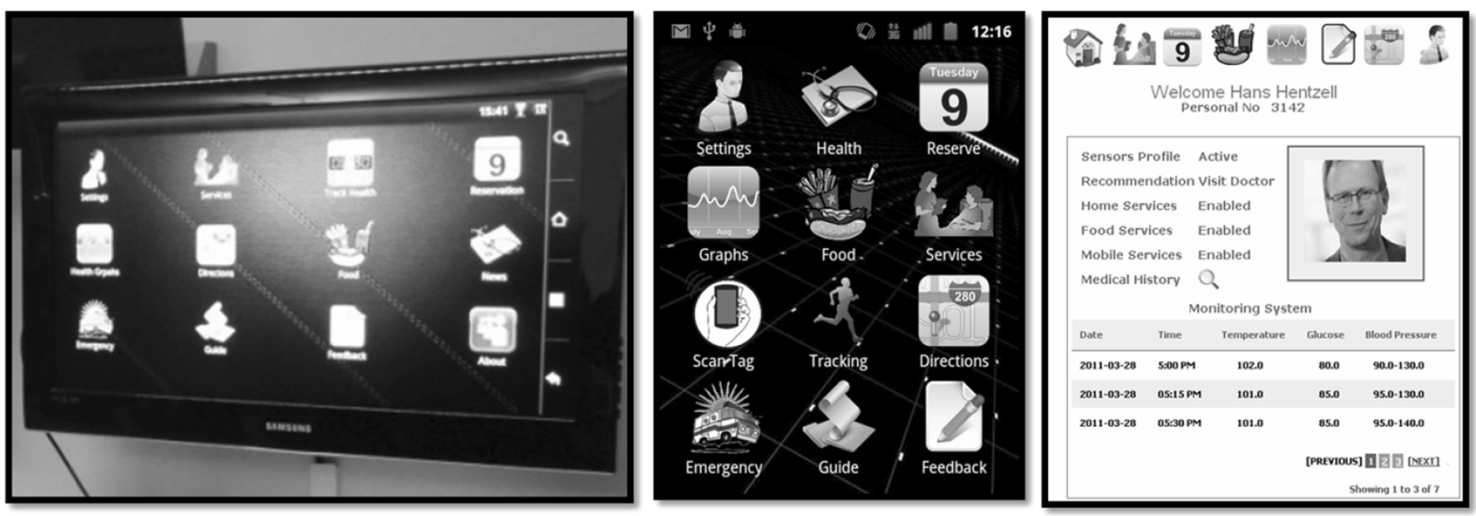

Fig. 9 The proof-of-concept application on a TV, a smartphone, and on the Web

increase as technology develops. But the need for balancing the resource consumptions against the available resources will still be a problem in future.

One cannot generally assume that as we spend more resources, the application quality will increase linearly well. This is because there exists a clear limit, which is based on our abilities as human beings. Where the quality of experience of an application does not increase beyond a certain threshold, just because we spend more resources. In detail, as we spend more and more resources, we will at some point hit this threshold where the quality of expedience no longer increases with the resource spending. For example, it is of no benefit to have lower response times beyond human recognition or to provide more search results than a human can manage. Thus, we will need to balance our resource consumptions based on the sought after quality of experience in our specific application scenario. There will be almost impossible to create a general resource model, because we cannot simply generalize over all types of scenarios. Thus, without considering the applications full situation, target group, and scenario, we can only provide indications for specific scenarios and provide guidelines for the general case. It is to this end we provide a potential way of balancing the resource consumption, by adapting, for example, the threshold value of the context relevance function from Eq. 3 back in Sect. 3.1

\section{Conclusions}

This paper presented a peer-to-peer-based architecture for sensor-assisted applications with real-time demands of the information dissemination on a global scale. The real-time demands are addressed with peer-to-peer-based communication that does not proxy or re-route any of the information. All information is exchanged directly between entities to reduce delay and to ensure that the information is received within the bounded time demands of real-time applications. Furthermore, the architecture is provided as an application platform and is lightweight enough to run on mobile devices with limited resources, which was also shown in a proof-of-concept application running on three different types of devices. Thus, we have shown that if we equip users with many different types of sensor systems connected to our architecture, it will be possible to create feature rich applications based on the users current contextual situation for a wide range of application. Our contribution beyond the already established platform has been to adopt a generic information model which is extensible and thus enables intelligent application behavior as well as enabling features such as persistent storage and offloading computational heavy tasks. Our evaluation indicates that our proposed architecture should be more reliable and well scaling than centralized and semi-distributed solutions, while still being lightweight when compared to other fully distributed architectures. From this, we can also conclude that we can successfully address all the concrete goals stated in Sect. 1 .

Goal 1 on seamless access to global sensor information in a ubiquitous manner was achieved with our globally spanning platform, which allows any entity to communicate with any other entity which is connected to the platform. Goal 2 on real-time sharing of sensor information was addressed by applying a peer-to-peer-based exchange of information, with low delays and no unnecessary proxies. Goal 3 on scaling for a vast number of connected entities was also achieved with our peer-to-peer-based approach. This is because our system scales logarithmically with the amount of connected entities, and it avoids any central points of failure. Goal 4 on having a generic information model which enables intelligent application behavior was addressed with our relevance based context schemes, which represent an entity's current situation. Goal 5 on the support for discovery, querying, and searching for new sources of information was addressed by performing relevance and ranking on the context schemes, 
both locally on end devices and in powerful cloud-based support systems. Goal 6 on the ability to balance the resource consumptions against quality-of-experience requirements was achieved by allowing the end devices and applications themselves to control the amount communication and computational power they are willing to spend on resource consuming tasks.

Our current work is directed toward further evaluating resource consumptions based on quality of experience. This in order to determine when an application is considered being good enough and any further resources spent are being wasted. Future work also includes further studies into a potential impending information overload on the Internetof-things and the impact of continuously changing context information on end devices, especially toward the managing of resource limitations based on different application demands.

Acknowledgments This work has been supported by Grant 2010-00681 of VINNOVA the Swedish Governmental Agency for Innovation Systems and by Grant 00163383 of the EU European Regional Development Fund, Mellersta Norrland, Sweden.

\section{References}

1. Hong J, Suh E, Kim S (2009) Context-aware systems: a literature review and classification. Expert Syst Appl 36(4):8509-8522

2. Atzori L, Iera A, Morabito G (2010) The internet of things: a survey. Comput Netw 54(15):2787-2805

3. Ericsson (2011) More than 50 billion connected devices. White Paper. [Online]. Available: http://www.ericsson.com/res/docs/ whitepapers/wp-50-billions.pdf

4. Bricon-Souf N, Newman C (2007) Context awareness in health care: a review. Int J Med Inform 76(1):2-12

5. Chan M, Campo E, Estève D, Fourniols J (2009) Smart homescurrent features and future perspectives. Maturitas 64(2):90-97

6. Bellavista P, Kupper A, Helal S (2008) Location-based services: back to the future. Pervasive Comput IEEE 7(2):85-89

7. Kansal A, Nath S, Liu J, Zhao F (2007) Senseweb: an infrastructure for shared sensing. IEEE MultiMed 14(4):8-13

8. Presser M, Barnaghi P, Eurich M, Villalonga C (2009) The SENSEI project: integrating the physical world with the digital world of the network of the future. Commun Mag IEEE 47(4): $1-4$

9. SicsthSense. [Online]. Available: http://sense.sics.se

10. Klemettinen M (2007) Enabling technologies for mobile services: the mobilife book. John Wiley and Sons, Chichester

11. Raz D, Juhola A, Serrat-Fernandez J, Galis A (2006) Fast and efficient context-aware services. John Wiley and Sons, Chichester

12. Koumaras H, Negrou D, Liberal F, Arauz J, Kourtis A (2008) ADAMANTIUM project: enhancing IMS with a PQoS-aware multimedia content management system. Int Conf Autom Qual Test Robot 1:358-363

13. Rosenberg J, Schulzrinne H, Camarillo G, Johnston A, Peterson J, Sparks R, Handley M, Schooler E (2002) SIP: Session Initiation Protocol. Network Working Group, RFC 3261, June

14. Toninelli A, Pantsar-Syväniemi S, Bellavista P, Ovaska E (2009) Supporting context awareness in smart environments: a scalable approach to information interoperability. In: Proceedings of the international workshop on middleware for pervasive mobile and embedded computing. ACM, pp 1-4

15. Bellavista P, Montanari R, Tibaldi D (2003) Cosmos: a contextcentric access control middleware for mobile environments. In: Mobile agents for telecommunication applications. Springer, Berlin, pp 77-88

16. Walters J (2011) Ripples across the internet of things: context metrics as vehicles for relational self-organization. Mid Sweden University, Sundsvall

17. Nitti M, Girau R, Atzori L, Iera A, Morabito G (2012) A subjective model for trustworthiness evaluation in the social internet of things. In: International workshop on internet-of-things communications and networking (IoT-CN)

18. Kanter T, Österberg P, Walters J, Kardeby V, Forsström S, Pettersson S (2009) The mediasense framework. In: Proceedings of 4th IARIA international conference on digital telecommunications (ICDT). Colmar, July, pp 144-147

19. Kanter T, Forsström S, Kardeby V, Walters J, Jennehag U, Österberg P (2012) Mediasense-an internet of things platform for scalable and decentralized context sharing and control. In: Proceedings of 7th IARIA international conference on digital telecommunications (ICDT).

20. Kanter T, Pettersson S, Forsstrom S, Kardeby V, Norling R, Walters J, Osterberg P (Aug 2009) Distributed context support for ubiquitous mobile awareness services. In: Fourth International Conference on Communications and networking in China, 2009. ChinaCOM 2009, pp 1-5

21. Stoica I, Morris R, Karger D, Kaashoek F, Balakrishnan H (2001) Chord: a scalable peer-to-peer lookup service for internet applications. In: Proceedings of the conference on applications, technologies, architectures, and protocols for computer communications, vol 31. ACM Press, New York, pp 149-160 